\title{
Spectral Approximation for Compact Operators
}

\author{
By John E. Osborn*
}

\begin{abstract}
In this paper a general spectral approximation theory is developed for compact operators on a Banach space. Results are obtained on the approximation of eigenvalues and generalized eigenvectors. These results are applied in a variety of situations.
\end{abstract}

1. Introduction. Recently, Bramble and Osborn [7] developed spectral approximation results for a particular type of compact operator on a Hilbert space and applied them to obtain rate of convergence estimates for several Galerkin-type approximations for the eigenvalues and generalized eigenvectors of nonselfadjoint elliptic partial differential operators.

In this paper spectral approximation results are established for compact operators on a Banach space. These results are then applied in a variety of situations: Galerkintype approximations for nonselfadjoint elliptic operators, approximation of integral operators by numerical quadrature, and an approximation by "homogenization" recently studied by Babuška [4], [5].

The main results of the paper are in Section 3. The development there is strongly influenced by the treatment in [7]. The results in [7] are formulated in terms of Sobolev space norms of negative as well as positive order, whereas the results in this paper are formulated just in terms of the norm on the underlying Banach space. Special features of this work are:

1. Approximations for the generalized eigenvectors are obtained first and then used to obtain the eigenvalue estimates.

2. In the case of a multiple eigenvalue (in general having different algebraic and geometric multiplicities), a weighted average of approximate eigenvalues is shown to be the "right" choice as an approximation.

3. The results apply to any compact operator on a Banach space and thus, in particular, to nonselfadjoint operators on a Hilbert space.

4. The results apply to a variety of approximation methods such as Galerkin-type approximation of elliptic eigenvalue problems and the approximation of integral operators by numerical quadrature.

Our results should be compared to the work of Vainnikko [18], [19], [20]. He proves a general theorem on the convergence of eigenvalues and generalized eigenvectors. Theorems 4 and 5 of Section 3 are analogous to his. He does not obtain a result of the type given by Theorem 3 of Section 3 (our main result). His strongest results are

Received March 18, 1974.

AMS (MOS) subject classifications (1970). Primary 35P15, 65N15, 65N25, 65N30; Secondary $15 \mathrm{~A} 18,47 \mathrm{~A} 15$.

Key words and phrases. Approximation of eigenvalues, approximation of generalized eigenvectors, nonselfadjoint operators, finite element method.

*Partially supported by NSF Grant GP 35666. 
associated with the approximation of an operator $T$ by an operator $P_{n} T$ where $P_{n}$ is a projection whose adjoint can be calculated (cf. part (d) of Section 4). This calculation, which must be carried out for each application, does not appear to be simple and it is not clear how to put several of the applications mentioned in Section 4 into Vainikko's setting.

2. Preliminaries. Let $A: X \rightarrow X$ be a compact operator on a complex Banach space $X$. We denote by $\sigma(A)$ and $\rho(A)$ the spectrum and resolvent sets of $A$, respectively. For any complex number $z \in \rho(A), R_{z}(A)=(z-A)^{-1}$ is the resolvent operator. $\sigma(A)$ is countable; nonzero numbers in $\sigma(A)$ are eigenvalues; and if zero is in $\sigma(A)$, it may or may not be an eigenvalue.

Let $\mu \in \sigma(A)$ be nonzero. There is a smallest integer $\alpha$ such that $N\left((\mu-A)^{\alpha}\right)=$ $N\left((\mu-A)^{\alpha+1}\right)$, where $N$ denotes the null space; $\alpha$ is called the ascent of $\mu-A$. $N\left((\mu-A)^{\alpha}\right)$ is finite dimensional and $m=\operatorname{dim} N\left((\mu-A)^{\alpha}\right)$ is called the algebraic multiplicity of $\mu$. The vectors in $N\left((\mu-A)^{\alpha}\right)$ are called the generalized eigenvectors of $A$ corresponding to $\mu$. The geometric multiplicity of $\mu$ is equal to $\operatorname{dim} N(\mu-A)$ and is less then or equal to the algebraic multiplicity. The two multiplicities are equal if $X$ is a Hilbert space and $A$ is selfadjoint.

Throughout the paper we will consider a compact operator $T: X \rightarrow X$ and a sequence of compact operators $T_{n}: X \rightarrow X$ such that $T_{n} \rightarrow T$ pointwise $\left(\lim _{n \rightarrow \infty}\left\|T_{n} f-T f\right\|=0\right.$ for all $\left.f \in X\right)$ and $\left\{T_{n}\right\}$ is collectively compact, i.e., the set $\left\{T_{n} f:\|f\| \leqslant 1, n=1,2, \ldots\right\}$ is sequentially compact. Clearly this is the case if $T_{n} \rightarrow T$ in norm $\left(\lim _{n \rightarrow \infty}\left\|T_{n}-T\right\|=0\right)$.

Let $\mu$ be a nonzero eigenvalue of $T$ with algebraic multiplicity $m$ and let $\Gamma$ be a circle centered at $\mu$ which lies in $\rho(T)$ and which encloses no other points of $\sigma(T)$. The spectral projection associated with $\mu$ and $T$ is defined by

$$
E=E(\mu)=\frac{1}{2 \pi i} \int_{\Gamma} R_{z}(T) d z .
$$

$E$ is a projection onto the space of generalized eigenvectors associated with $\mu$ and $T$. For $n$ sufficiently large, $\Gamma \subset \rho\left(T_{n}\right)$ and the spectral projection,

$$
E_{n}=E_{n}(\mu)=\frac{1}{2 \pi i} \int_{\Gamma} R_{z}\left(T_{n}\right) d z
$$

exists; $E_{n}$ converges to $E$ pointwise and $\left\{E_{n}\right\}$ is collectively compact; and $\operatorname{dim} R\left(E_{n}(\mu)\right)$ $=\operatorname{dim} R(E(\mu))=m$, where $R$ denotes the range. $E_{n}$ is the spectral projection associated with $T_{n}$ and the eigenvalues of $T_{n}$ which lie in $\Gamma$, and is a projection onto the direct sum of the spaces of generalized eigenvectors corresponding to these eigenvalues. Thus, counting according to algebraic multiplicities, there are $m$ eigenvalues of $T_{n}$ in $\Gamma$; we denote these by $\mu_{1}(n), \ldots, \mu_{m}(n)$. Furthermore, if $\Gamma^{\prime}$ is another circle centered at $\mu$ with an arbitrarily small radius, we see that $\mu_{1}(n), \ldots, \mu_{m}(n)$ are all inside of $\Gamma^{\prime}$ for $n$ sufficiently large, i.e., $\lim _{n \rightarrow \infty} \mu_{j}(n)=\mu$ for $j=1, \ldots, m$. $R(E)$ and $R\left(E_{n}\right)$ are invariant subspaces for $T$ and $T_{n}$, respectively, and $T E=E T$ and $T_{n} E_{n}=E_{n} T_{n}$. We will also use the fact that $\left\{R_{z}\left(T_{n}\right): z \in \Gamma, n\right.$ large $\}$ is bounded.

In the context considered here, namely, pointwise convergence of a sequence of collectively compact operators, these results can all be found in Anselone [1]. For the 
case of norm convergence, they can be found in [9].

If $\mu$ is an eigenvalue of $T$ with algebraic multiplicity $m$, then $\mu$ is an eigenvalue with algebraic multiplicity $m$ of the adjoint operator $T^{*}$ on the dual space $X^{*}$. The ascent of $\mu-T^{*}$ will be $\alpha . E^{*}$ will be the projection operator associated with $T^{*}$ and $u$; likewise $E_{n}^{*}$ will be the projection operator associated with $T_{n}^{*}$ and $\mu_{1}(n), \ldots, \mu_{m}(n)$. If $\phi \in X$ and $\phi^{*} \in X^{*}$, we will denote the value of the linear functional $\phi^{*}$ at $\phi$ by $\left[\phi, \phi^{*}\right]$.

Remark. $T^{*}$ here is the Banach adjoint. If $X=H$ is a Hilbert space; we would naturally work with the Hilbert adjoint, which acts on $H$. Then $\mu$ would be an eigenvalue of $T$ if and only if $\bar{\mu}$ is an eigenvalue of $T^{*}$.

Given two closed subspaces $M$ and $N$ of $X$ we define $\delta(M, N)=$ $\sup _{x \in M,\|x\|=1} \operatorname{dist}(x, N)$ and $\hat{\delta}(M, N)=\max [\delta(M, N), \delta(N, M)] ; \hat{\delta}(M, N)$ is called the gap between $M$ and $N$. The gap provides a natural way in which to formulate results on the approximation of generalized eigenvectors. We will need the following results.

LEMma 1. If $\operatorname{dim} M=\operatorname{dim} N<\infty$, then

$$
\delta(N, M) \leqslant \delta(M, N)[1-\delta(M, N)]^{-1} .
$$

For a discussion of this result and the result that $\delta(N, M)=\delta(M, N)$ if $X=H$ is a Hilbert space and $\hat{\delta}(N, M)<1$, we refer to [10, pp. 264-269].

LEMMA 2 (LAY [13]). Let $N, M_{1}$ and $M_{2}$ be closed subspaces of $X$ such that $X=M_{1} \oplus N=M_{2} \oplus N$ and let $Q_{1}$ and $Q_{2}$ be the projections of $X$ along $N$ and onto $M_{1}$ and $M_{2}$, respectively. If $\left\|I-Q_{2}\right\| \delta\left(M_{1}, M_{2}\right)<1$, then

$$
\left\|Q_{1}-Q_{2}\right\| \leqslant \frac{\left\|Q_{2}\right\|\left\|I-Q_{2}\right\| \delta\left(M_{1}, M_{2}\right)}{1-\left\|I-Q_{2}\right\| \delta\left(M_{1}, M_{2}\right)}
$$

Proof. Let $d=\sup _{x \in M_{1},\|x\|=1}\left\|x-Q_{2} x\right\|$. Let $\delta>\delta\left(M_{1}, M_{2}\right), x \in M_{1},\|x\|=$ 1. Then by the definition of $\delta\left(M_{1}, M_{2}\right)$ there exists $u \in M_{2}$ such that $\|x-u\|<\delta$. Since $\left(I-Q_{2}\right) u=0$, we have $\left\|x-Q_{2} x\right\|=\left\|\left(I-Q_{2}\right)(x-u)\right\|<\left\|I-Q_{2}\right\| \delta$. Thus $d \leqslant$ $\left\|I-Q_{2}\right\| \delta$ for all $\delta>\delta\left(M_{1}, M_{2}\right)$ and hence $d \leqslant\left\|I-Q_{2}\right\| \delta\left(M_{1}, M_{2}\right)$.

Since $R\left(I-Q_{1}\right)=N\left(Q_{2}\right)$, we have $Q_{2}=Q_{2} Q_{1}$. It is clear that $\left\|y-Q_{2} y\right\| \leqslant$ $d\|y\|$ for all $y \in M_{1}$. Hence for any $x$,

$$
\left\|Q_{1} x\right\| \leqslant\left\|Q_{1} x-Q_{2} Q_{1} x\right\|+\left\|Q_{2} Q_{1} x\right\| \leqslant d\left\|Q_{1} x\right\|+\left\|Q_{2} x\right\|,
$$

from which we get $\left\|Q_{1} x\right\| \leqslant(1-d)^{-1}\left\|Q_{2} x\right\|$. Thus, since $d \leqslant\left\|I-Q_{2}\right\| \delta\left(M_{1}, M_{2}\right)<1$, we have

$$
\left\|Q_{1} x-Q_{2} x\right\|=\left\|Q_{1} x-Q_{2} Q_{1} x\right\| \leqslant d\left\|Q_{1} x\right\| \leqslant \frac{\left\|I-Q_{2}\right\|\left\|Q_{2}\right\| \delta\left(M_{1}, M_{2}\right)}{1-\left\|I-Q_{2}\right\| \delta\left(M_{1}, M_{2}\right)} .
$$

3. Convergence Estimates. Let $\mu$ be a nonzero eigenvalue of $T$ and assume the ascent of $\mu-T$ is $\alpha$. We begin this section by showing how the generalized eigenvectors of $T$ corresponding to $\mu$ are approximated by the generalized eigenvectors of $T_{n}$ corresponding to $\mu_{1}(n), \ldots, \mu_{m}(n)$.

THEOREM 1. There is a constant $C_{1}$ such that 


$$
\hat{\delta}\left(R(E), R\left(E_{n}\right)\right) \leqslant C_{1}\left\|\left.\left(T-T_{n}\right)\right|_{R(E)}\right\|
$$

for all large $n$, where $\left.\left(T-T_{n}\right)\right|_{R(E)}$ denotes the restriction of $T-T_{n}$ to $R(E)$.

Proof. For $f \in R(E)$ with $\|f\|=1$, we have $\left\|f-E_{n} f\right\|=\left\|\left(E-E_{n}\right) E f\right\| \leqslant$ $\left\|\left(E-E_{n}\right) E\right\|$, and hence $\delta\left(R(E), R\left(E_{n}\right)\right) \leqslant\left\|\left(E-E_{n}\right) E\right\| ;$ for $f \in R\left(E_{n}\right)$ with $\|f\|=1$ we have $\|f-E f\| \leqslant\left\|\left(E_{n}-E\right) E_{n}\right\|$, and hence $\delta\left(R\left(E_{n}\right), R(E)\right) \leqslant\left\|\left(E_{n}-E\right) E_{n}\right\|$. Since $E_{n} \rightarrow E$ pointwise and $\left\{E_{n}\right\}$ is collectively compact, both $\left\|\left(E_{n}-E\right) E\right\|$ and $\left\|\left(E-E_{n}\right) E_{n}\right\|$ converge to 0 as $n \rightarrow \infty$ [1]. Thus $\lim _{n \rightarrow \infty} \delta\left(R(E), R\left(E_{n}\right)\right)=0$.

We now apply Lemma 1 with $M=R(E)$ and $N=R\left(E_{n}\right)$. This yields

$$
\delta\left(R\left(E_{n}\right), R(E)\right) \leqslant \delta\left(R(E), R\left(E_{n}\right)\right)\left[1-\delta\left(R(E), R\left(E_{n}\right)\right)\right]^{-1} .
$$

Since $\lim _{n+\infty} \hat{\delta}\left(R(E), R\left(E_{n}\right)\right)=0,(3.1)$ implies that $\delta\left(R\left(E_{n}\right), R(E)\right) \leqslant C \delta\left(R(E), R\left(E_{n}\right)\right)$ for some constant $C$ and hence that

$$
\delta\left(R\left(E_{n}\right), R(E)\right) \leqslant(1+C) \delta\left(R(E), R\left(E_{n}\right)\right) .
$$

Now for $f \in R(E)$ we have

$$
\begin{aligned}
\left\|f-E_{n} f\right\| & =\left\|E f-E_{n} f\right\|=\left\|\frac{1}{2 \pi i} \int_{\Gamma}\left[R_{z}(T)-R_{z}\left(T_{n}\right)\right] f d z\right\| \\
& =\frac{1}{2 \pi}\left\|\int_{\Gamma} R_{z}\left(T_{n}\right)\left(T-T_{n}\right) R_{z}(T) f d z\right\| .
\end{aligned}
$$

$R(E)$ is invariant for $T$ and thus for $R_{z}(T)$. Hence

$$
\left\|f-E_{n} f\right\| \leqslant \frac{1}{2 \pi} \text { length }(\Gamma) \sup _{z \in \Gamma}\left\|R_{z}\left(T_{n}\right)\right\|\left\|\left.\left(T-T_{n}\right)\right|_{R(E)}\right\| \sup _{z \in \Gamma}\left\|R_{z}(T)\right\|\|f\| .
$$

Since $T_{n} \rightarrow T$ pointwise and $\left\{T_{n}\right\}$ is collectively compact, $\sup _{z \in \Gamma}\left\|R_{z}\left(T_{n}\right)\right\|$ is bounded in $n$. Thus from (3.2) and (3.3) we have

where

$$
\hat{\delta}\left(R(E), R\left(E_{n}\right)\right) \leqslant C_{1}\left\|\left.\left(T-T_{n}\right)\right|_{R(E)}\right\|,
$$

$$
C_{1}=(1 / 2 \pi)(1+C) \text { length }(\Gamma) \sup _{n, z \in \Gamma}\left\|R_{z}\left(T_{n}\right)\right\| \sup _{z \in \Gamma}\left\|R_{z}(T)\right\| .
$$

Although each of the eigenvalues $\mu_{1}(n), \ldots, \mu_{m}(n)$ are close to $\mu$ for large $n$, their arithmetic mean is generally a closer approximation [7]. Thus we define

$$
\hat{\mu}(n)=\frac{1}{m} \sum_{j=1}^{m} \mu_{j}(n) .
$$

In the terminology of [11] this is the weighted mean of the $\mu$-group. See also Kreiss [12]. Our next theorem gives an estimate for $\mu-\hat{\mu}(n)$.

THEOREM 2. There is a constant $C_{2}$ such that

$$
|\mu-\hat{\mu}(n)| \leqslant C_{2}\left\|\left.\left(T-T_{n}\right)\right|_{R(E)}\right\|
$$

for all large $n$.

Proof. For large $n$ the operator $\left.E_{n}\right|_{R(E)}: R(E) \rightarrow R\left(E_{n}\right)$ is one-to-one since $\left\|\left(E-E_{n}\right) E\right\| \rightarrow 0$ and $E_{n} f=0, f \in R(E)$ implies $\|f\|=\left\|E f-E_{n} f\right\| \leqslant\left\|\left(E-E_{n}\right) E\right\| \cdot$ 
$\|f\|$, and $\left.E_{n}\right|_{R(E)}$ is onto, since $\operatorname{dim} R\left(E_{n}\right)=\operatorname{dim} R(E)=m$. Thus $\left(\left.E_{n}\right|_{R(E)}\right)^{-1}$ :

$R\left(E_{n}\right) \rightarrow R(E)$ is defined; we write $E_{n}^{-1}$ for $\left(\left.E_{n}\right|_{R(E)}\right)^{-1}$. For $n$ sufficiently large and $f \in R(E)$ with $\|f\|=1$, we have

$$
1-\left\|E_{n} f\right\|=\|E f\|-\left\|E_{n} f\right\| \leqslant\left\|\left(E-E_{n}\right) E\right\| \leqslant 1 / 2
$$

and hence $\left\|E_{n} f\right\| \geqslant 1 / 2$. This implies $\left\|E_{n}^{-1}\right\| \leqslant 2$ for large $n$, i.e., $\left\|E_{n}^{-1}\right\|$ is bounded in $n$. We note that $E_{n} E_{n}^{-1}$ is the identity on $R\left(E_{n}\right)$ and $E_{n}^{-1} E_{n}$ is the identity on $R(E)$. Now we define $\hat{T}=\left.E_{n}^{-1} T_{n} E_{n}\right|_{R(E)}: R(E) \rightarrow R(E)$.

Using the fact that $R\left(E_{n}\right)$ is invariant for $T_{n}$, we see that $\sigma\left(\hat{T}_{n}\right)=\left\{\mu_{1}(n), \ldots\right.$, $\mu_{m}(n)$ \} and that the algebraic [geometric] multiplicity of any $\mu_{j}(n)$ as an eigenvalue of $\hat{T}_{n}$ is equal to its algebraic [geometric] multiplicity as an eigenvalue of $T_{n}$. Letting $\hat{T}=\left.T\right|_{R(E)}$ we likewise see that $\sigma(\hat{T})=\{\mu\}$. Thus trace $\hat{T}=m \mu$ and trace $\hat{T}_{n}=$ $m \hat{\mu}(n)$, and since $\hat{T}$ and $\hat{T}_{n}$ are defined on the same space, we have

$$
\mu-\hat{\mu}(n)=\frac{1}{m} \operatorname{trace}\left(\hat{T}-\hat{T}_{n}\right) .
$$

The use of the operator $\hat{T}_{n}$ was suggested by Atkinson [2].

Let $\phi_{1}, \ldots, \phi_{m}$ be a basis for $R(E)$ and let $\phi_{1}^{*}, \ldots, \phi_{m}^{*}$ be the dual basis to $\phi_{1}, \ldots, \phi_{m}$. Then

$$
\mu-\hat{\mu}(n)=\frac{1}{m} \operatorname{trace}\left(\hat{T}-\hat{T}_{n}\right)=\frac{1}{m} \sum_{j=1}^{m}\left[\left(\hat{T}-\hat{T}_{n}\right) \phi_{j}, \phi_{j}^{*}\right] .
$$

Here each $\phi_{j}^{*}$ is an element of $R(E)^{*}$, the dual space of $R(E)$, but we may extend each $\phi_{j}^{*}$ to $X$ as follows. Since $X=R(E) \oplus N(E)$, any $f \in X$ can be written as $f=g+h$ with $g \in R(E)$ and $h \in N(E)$. Define $\left[f, \phi_{j}^{*}\right]=\left[g, \phi_{j}^{*}\right]$. Clearly $\phi_{j}^{*}$, so extended, is bounded, i.e., $\phi_{j}^{*} \in X^{*}$. Now $\left[f,\left(\mu-T^{*}\right)^{\alpha} \phi_{j}^{*}\right]=\left[(\mu-T)^{\alpha} f, \phi_{j}^{*}\right]$. If $f \in R(E)=$ $N\left((\mu-T)^{\alpha}\right)$, this vanishes and if $f \in N(E)$, this vanishes since $N(E)$ is invariant for $\mu-$ $T$. Hence $\left[f,\left(\mu-T^{*}\right)^{\alpha} \phi_{j}^{*}\right]=0$ for all $f \in X$, i.e., $\left(\mu-T^{*}\right)^{\alpha} \phi_{j}^{*}=0$. Thus we have shown that the $\phi_{j}^{*}$ are generalized eigenvectors of $T^{*}$ corresponding to $\mu$. Although this particular choice for the extension of $\phi_{j}^{*}$ plays no role in this theorem, it is central in the theorems which follow.

Using the facts that $T_{n} E_{n}=E_{n} T_{n}$ and $E_{n}^{-1} E_{n}$ is the identity on $R(E)$, we see that

$$
\begin{aligned}
& \left|\left[\left(T-T_{n}\right) \phi_{j}, \phi_{j}^{*}\right]\right|=\left|\left[T \phi_{j}-E_{n}^{-1} T_{n} E_{n} \phi_{j}, \phi_{j}^{*}\right]\right| \\
& \quad=\left|\left[E_{n}^{-1} E_{n}\left(T-T_{n}\right) \phi_{j}, \phi_{j}^{*}\right]\right| \leqslant\left\|E_{n}^{-1} E_{n}\right\|\left\|\left.\left(T-T_{n}\right)\right|_{R(E)}\right\|\left\|\phi_{j}\right\|\left\|\phi_{j}^{*}\right\|
\end{aligned}
$$

for each $j$. $\left\|E_{n}\right\|$ is bounded in $n$ since $E_{n} \rightarrow E$ pointwise. Thus from (3.4) and (3.5) we get

$$
|\mu-\hat{\mu}(n)| \leqslant \frac{1}{m} \sup _{k}\left\|E_{k}^{-1} E_{k}\right\| \sum_{j=1}^{m}\left\|\phi_{j}\right\|\left\|\phi_{j}^{*}\right\|\left\|\left.\left(T-T_{n}\right)\right|_{R(E)}\right\| .
$$

This gives the desired result.

For the remainder of the paper, except where the contrary is explicitly pointed out, we assume $T_{n}$ converges to $T$ in norm. Then $T_{n}^{*}$ converges to $T^{*}$ in norm. Under the assumption of norm convergence we can obtain a refined estimate for $|\mu-\hat{\mu}(n)|$.

THEOREM 3. Let $\phi_{1}, \ldots, \phi_{m}$ be any basis for $R(E)$ and let $\phi_{1}^{*}, \ldots, \phi_{m}^{*}$ be the dual basis (chosen as in the proof of Theorem 2). Then there is a constant $C_{3}$ such that 


$$
|\mu-\hat{\mu}(n)| \leqslant \frac{1}{m} \sum_{j=1}^{m}\left|\left[\left(T-T_{n}\right) \phi_{j}, \phi_{j}^{*}\right]\right|+C_{3}\left\|\left.\left(T-T_{n}\right)\right|_{R(E)}\right\|\left\|\left.\left(T^{*}-T_{n}^{*}\right)\right|_{R\left(E^{*}\right)}\right\| .
$$

Proof. From (3.5) we have

$$
\begin{aligned}
{\left[\left(\hat{T}-\hat{T}_{n}\right) \phi_{j}, \phi_{j}^{*}\right] } & =\left[\left(E_{n}^{-1} E_{n}\right)\left(T-T_{n}\right) \phi_{j}, \phi_{j}^{*}\right] \\
& =\left[\left(T-T_{n}\right) \phi_{j},\left(E_{n}^{-1} E_{n}\right)^{*} \phi_{j}^{*}\right] \\
& =\left[\left(T-T_{n}\right) \phi_{j}, \phi_{j}^{*}\right]+\left[\left(T-T_{n}\right) \phi_{j},\left(E_{n}^{-1} E_{n}\right)^{*} \phi_{j}^{*}-\phi_{j}^{*}\right] .
\end{aligned}
$$

Let $L_{n}=E_{n}^{-1} E_{n} . L_{n}$ is the projection on $R(E)$ along $N\left(E_{n}\right)$. Thus $L_{n}^{*}$ is the projection on $N\left(E_{n}\right)^{\perp}=R\left(E_{n}^{*}\right)$ along $R(E)^{\perp}=N\left(E^{*}\right)$. Since, as shown in the proof of Theorem 2, $\phi_{j}^{*} \in N\left(\left(\mu-T^{*}\right)^{\alpha}\right)=R\left(E^{*}\right)$ we have $L_{n}^{*} \phi_{j}^{*}-\phi_{j}^{*}=\left(L_{n}^{*}-E^{*}\right) \phi_{j}^{*}$. Thus from Lemma 2 with $N=N\left(E^{*}\right), M_{1}=R\left(E_{n}^{*}\right)$ and $M_{2}=R\left(E^{*}\right)$ as subspaces of $X^{*}$,

$$
\left\|L_{n}^{*} \phi_{j}^{*}-\phi_{j}^{*}\right\| \leqslant \frac{\left\|E^{*}\right\|\left\|I-E^{*}\right\| \hat{\delta}\left(R\left(E^{*}\right), R\left(E_{n}^{*}\right)\right)\left\|\phi_{j}^{*}\right\|}{1-\left\|I-E^{*}\right\| \hat{\delta}\left(R\left(E^{*}\right), R\left(E_{n}^{*}\right)\right)} .
$$

Note that Theorem 1 (applied to $T^{*}$ and $\left.\left\{T_{n}^{*}\right\}\right)$ implies that $\lim _{n \rightarrow \infty} \delta\left(R\left(E^{*}\right), R\left(E_{n}^{*}\right)\right)=$ 0 , and hence Lemma 2 is applicable. From (3.7) and Theorem 1 (again applied to $T^{*}$ and $\left.\left\{T_{n}^{*}\right\}\right)$, we have

$$
\left\|L_{n}^{*} \phi_{j}^{*}-\phi_{j}^{*}\right\| \leqslant C_{3}\left\|\left.\left(T^{*}-T_{n}^{*}\right)\right|_{R\left(E^{*}\right)}\right\|
$$

for some constant $C_{3}$. Now, combining (3.4), (3.6) and (3.8), we obtain

$$
|\mu-\hat{\mu}(n)| \leqslant \frac{1}{m} \sum_{j=1}^{m}\left|\left[\left(T-T_{n}\right) \phi_{j}, \phi_{j}^{*}\right]\right|+C_{3}\left\|\left.\left(T-T_{n}\right)\right|_{R(E)}\right\|\left\|\left.\left(T^{*}-T_{n}^{*}\right)\right|_{R\left(E^{*}\right)}\right\| .
$$

In addition to estimating $\mu-\hat{\mu}(n)$ we may estimate $\mu-\mu_{j}(n)$ for each $j$.

THEOREM 4. Let $\alpha$ be the ascent of $\mu-T$. Let $\phi_{1}, \ldots, \phi_{m}$ be a basis for $R(E)$, and let $\phi_{1}^{*}, \ldots, \phi_{m}^{*}$ be the dual basis. Then there is a constant $C_{4}$ such that

$$
\left|\mu-\mu_{j}(n)\right|^{\alpha} \leqslant C_{4}\left\{\sum_{i, j=1}^{m}\left|\left[\left(T-T_{n}\right) \phi_{i}, \phi_{j}^{*}\right]\right|+\left\|\left.\left(T-T_{n}\right)\right|_{R(E)}\right\|\left\|\left.\left(T^{*}-T_{n}^{*}\right)\right|_{R\left(E^{*}\right)}\right\|\right\} \text {. }
$$

Proof. For each $n, \mu_{j}(n)$ is one of the eigenvalues of $\hat{T}_{n}$. Let $T_{n} w_{n}=\mu_{j}(n) w_{n}$, $\left\|w_{n}\right\|=1$. We can then choose $w_{n}^{*} \in N\left(\left(\mu-T^{*}\right)^{\alpha}\right)$ in such a way that $\left[w_{n}, w_{n}^{*}\right]=1$ and the norms $\left\|w_{n}^{*}\right\|$ are bounded. First, using the Hahn-Banach Theorem, choose $w_{n}^{*}$ $\in R(E)^{*}$ such that $\left[w_{n}, w_{n}^{*}\right]=1$ and $\left\|w_{n}^{*}\right\|=1$ and then extend $w_{n}^{*}$ to all of $X$ as in the proof of Theorem 2. $w_{n}^{*}$, so extended, will be in $R\left(E^{*}\right)$ and satisfy $\left\|w_{n}^{*}\right\| \leqslant\|E\|$. Now, noting that $(T-\mu)^{\alpha} w_{n}=0$, we have

$$
\begin{aligned}
\mid \mu- & \left.\mu_{j}(n)\right|^{\alpha}=\left|\left[\left(\mu-\mu_{j}(n)\right)^{\alpha} w_{n}, w_{n}^{*}\right]\right|=\left|\left[\left(\left(\mu-\mu_{j}(n)\right)^{\alpha}-(\mu-T)^{\alpha}\right) w_{n}, w_{n}^{*}\right]\right| \\
& =\left|\left[\sum_{j=0}^{\alpha-1}\left(\mu-\mu_{j}(n)\right)^{j}(\mu-T)^{\alpha-1-j}\left(\mu_{j}(n)-T\right) w_{n}, w_{n}^{*}\right]\right| \\
& \leqslant \sum_{j=0}^{\alpha-1}\left|\mu-\mu_{j}(n)\right|^{j}\left|\left[\left(\mu_{j}(n)-T\right) w_{n},\left(\mu-T^{*}\right)^{\alpha-1-j} w_{n}^{*}\right]\right| \\
& \leqslant \sum_{j=0}^{\alpha-1}\left|\mu-\mu_{j}(n)\right|_{\phi^{*} \in R\left(E^{*}\right),\left\|\phi^{*}\right\|=1}^{j}\left|\left[\left(\mu_{j}(n)-T\right) w_{n}, \phi^{*}\right]\right|\left\|\mu-T^{*}\right\|^{\alpha-1-j}\left\|w_{n}^{*}\right\| .
\end{aligned}
$$


For any $\phi^{*} \in R\left(E^{*}\right)$ with $\left\|\phi^{*}\right\|=1$,

$$
\begin{aligned}
& \left|\left[\left(\mu_{j}(n)-T\right) w_{n}, \phi^{*}\right]\right|=\left|\left[\left(\hat{T}_{n}-T\right) w_{n}, \phi^{*}\right]\right|=\left|\left[E_{n}^{-1} E_{n}\left(T_{n}-T\right) w_{n}, \phi^{*}\right]\right| \\
& \quad \leqslant\left|\left[\left(T_{n}-T\right) w_{n}, \phi^{*}\right]\right|+\left|\left[\left(T_{n}-T\right) w_{n}, L_{n}^{*} \phi^{*}-\phi^{*}\right]\right| \\
& \quad \leqslant\left|\left[\left(T_{n}-T\right) w_{n}, \phi^{*}\right]\right|+\left.C\left|\left(T-T_{n}\right)\right|_{R(E)}\|\|\left(T^{*}-T_{n}^{*}\right)\right|_{R\left(E^{*}\right)} \|
\end{aligned}
$$

There is a constant $C^{\prime}$ such that

$$
\left|\left[\left(T_{n}-T\right) w_{n}, \phi^{*}\right]\right| \leqslant C^{\prime} \sum_{i, j=1}^{m}\left|\left[\left(T_{n}-T\right) \phi_{i}, \phi_{j}^{*}\right]\right|
$$

for all $w_{n} \in R(E)$ and $\phi^{*} \in R\left(E^{*}\right)$ with $\left\|w_{n}\right\|=\left\|\phi^{*}\right\|=1$. Thus, using (3.9) and (3.10), we obtain

$$
\left|\mu-\mu_{j}(n)\right|^{\alpha} \leqslant C_{4}\left\{\sum_{i, j=1}^{m}\left|\left[\left(T-T_{n}\right) \phi_{i}, \phi_{j}^{*}\right]\right|+\left\|\left.\left(T-T_{n}\right)\right|_{R(E)}\right\|\left\|\left.\left(T^{*}-T_{n}^{*}\right)\right|_{R\left(E^{*}\right)}\right\|\right\} .
$$

Finally we consider the approximation of eigenvectors in $R(E)$ by eigenvectors in $R\left(E_{n}\right)$.

THEOREM 5. Let $\mu(n)$ be an eigenvalue of $T_{n}$ such that $\lim _{n \rightarrow \infty} \mu(n)=\mu$. Suppose for each $n$ that $w_{n}$ is a unit vector satisfying $\left(\mu(n)-T_{n}\right)^{k} w_{n}=0$ for some positive integer $k \leqslant \alpha$. Then, for any integer $l$ with $k \leqslant l \leqslant \alpha$, there is a vector $u_{n} \in$ $R(E)$ such that $(\mu-T)^{l} u_{n}=0$ and

$$
\left\|u_{n}-w_{n}\right\| \leqslant C_{5}\left\|\left.\left(T-T_{n}\right)\right|_{R(E)}\right\|^{(l-k+1) / \alpha} \text {. }
$$

Proof. Since $N\left((\mu-T)^{l}\right)$ is finite dimensional, there is a closed subspace $M$ of $X$ such that $X=N\left((\mu-T)^{l}\right) \oplus M$. For $y \in R\left((\mu-T)^{l}\right)$ the equation $(\mu-T)^{l} x=y$ is uniquely solvable in $M$. Thus $\left.(\mu-T)^{l}\right|_{M}: M \rightarrow R(\mu-T)^{l}$ is one-to-one and onto. Hence $\left.(\mu-T)^{l}\right|_{M} ^{-1}: R(\mu-T)^{l} \rightarrow M$ exists and by the closed graph theorem is bounded. Thus there is a constant $C$ such that $\|f\| \leqslant C\left\|(\mu-T)^{l} f\right\|$ for all $f \in M$.

Set $u_{n}=P w_{n}$ where $P$ is the projection on $N\left((\mu-T)^{l}\right)$ along $M$. Then $(\mu-T)^{l} u_{n}$ $=0$ and $w_{n}-u_{n} \in M$, and hence

$$
\left\|w_{n}-u_{n}\right\| \leqslant C\left\|(\mu-T)^{l}\left(w_{n}-u_{n}\right)\right\| .
$$

By Theorem 1 there are vectors $\tilde{u}_{n} \in R(E)$ such that

$$
\left\|w_{n}-\tilde{u}_{n}\right\| \leqslant C_{1}\left\|\left.\left(T-T_{n}\right)\right|_{R(E)}\right\| .
$$

Hence there is a constant $C^{\prime}$ such that

$$
\begin{aligned}
& \left\|\left[(\mu-T)^{l}-\left(\mu-T_{n}\right)^{l}\right] w_{n}\right\| \\
& \quad=\left\|\sum_{j=0}^{l-1}\left(\mu-T_{n}\right)^{j}\left(T-T_{n}\right)(\mu-T)^{l-j-1}\left[\left(w_{n}-\tilde{u}_{n}\right)+\tilde{u}_{n}\right]\right\| \\
& \quad \leqslant C^{\prime}\left\|\left.\left(T_{n}-T\right)\right|_{R(E)}\right\| .
\end{aligned}
$$

Since $k \leqslant l$, 


$$
\begin{aligned}
\left\|\left(\mu-T_{n}\right)^{l} w_{n}\right\| & =\left\|\sum_{j=0}^{l}\left(\begin{array}{l}
l \\
j
\end{array}\right)(\mu-\mu(n))^{j}\left(\mu(n)-T_{n}\right)^{l-j} w_{n}\right\| \\
& =\left\|\sum_{j=l-k+1}^{l}\left(\begin{array}{l}
l \\
j
\end{array}\right)(\mu-\mu(n))^{j}\left(\mu(n)-T_{n}\right)^{l-j} w_{n}\right\| \\
& \leqslant C^{\prime \prime}|\mu-\mu(n)|^{l-k+1}
\end{aligned}
$$

for some constant $C^{\prime \prime}$.

Now, combining (3.11), (3.12) and (3.13), we obtain

$$
\begin{aligned}
\left\|w_{n}-u_{n}\right\| & \leqslant C\left\|(\mu-T)^{l} w_{n}\right\| \\
& =C\left\|\left[(\mu-T)^{l}-\left(\mu-T_{n}\right)^{l}\right] w_{n}+(\mu-T)^{l} w_{n}\right\| \\
& \leqslant C\left\{C^{\prime}\left\|\left.\left(T-T_{n}\right)\right|_{R(E)}\right\|+C^{\prime \prime}|\mu-\mu(n)|^{l-k+1}\right\} .
\end{aligned}
$$

The result follows immediately from Theorem 4 .

We return now to the case where $T_{n} \rightarrow T$ pointwise and $\left\{T_{n}\right\}$ is collectively compact. Theorems 1 and 2 have been proved under this hypothesis and provide the main results on the approximation of generalized eigenvectors and eigenvalues, respectively. Corresponding to Theorem 4, we have the following

THEOREM 6. Suppose $T_{n} \rightarrow T$ pointwise and $\left\{T_{n}\right\}$ is collectively compact. Let $\alpha$ be the ascent of $\mu-T$. Then there is a constant $C_{6}$ such that

$$
\left|\mu-\mu_{j}(n)\right|^{\alpha} \leqslant C_{6}\left\|\left.\left(T-T_{n}\right)\right|_{R(E)}\right\| .
$$

Proof. Formula (3.9) in the proof of Theorem 4 is valid in this context. The result thus follows from the fact that

$$
\begin{aligned}
\left|\left[\left(\mu_{j}(n)-T\right) w_{n}, \phi^{*}\right]\right| & =\left|\left[E_{n}^{-1} E_{n}\left(T_{n}-T\right) w_{n}, \phi^{*}\right]\right| \\
& \leqslant \sup _{n}\left\|E_{n}^{-1} E_{n}\right\|\left\|\left.\left(T_{n}-T\right)\right|_{R(E)}\right\|
\end{aligned}
$$

for all $w_{n} \in R(E)$ and $\phi^{*} \in R\left(E^{*}\right)$ with $\left\|w_{n}\right\|=\left\|\phi^{*}\right\|=1$.

Finally we note that Theorem 5 is valid in this case also; the proof is exactly the same as that given for Theorem 5 except that at the end we use Theorem 6 instead of Theorem 4. Theorem 6 is essentially the same as the main result in Atkinson [2].

Thus we see that the eigenvector estimates are the same for norm convergence as for pointwise convergence of a collectively compact sequence but the eigenvalue estimates differ. The appearance of the expression $\left\|\left.\left(T-T_{n}\right)\right|_{R(E)}\right\|\left\|\left.\left(T^{*}-T_{n}^{*}\right)\right|_{R(E)}\right\|$ in the estimates in Theorems 3 and 4 as opposed to the expression $\left\|\left.\left(T-T_{n}\right)\right|_{R(E)}\right\|$ in Theorems 2 and 6 is a consequence of the fact that $\left\|T-T_{n}\right\| \rightarrow 0$ implies $\left\|T^{*}-T_{n}^{*}\right\|$ $\rightarrow 0$. If $T_{n} \rightarrow T$ pointwise and $\left\{T_{n}\right\}$ is collectively compact, it may happen that $T_{n}^{*}$ does not converge to $T^{*}$ pointwise and that $\left\{T_{n}^{*}\right\}$ is not collectively compact (cf. [1]). We note also that Theorems 3 and 4 hold under the assumption that $T_{n} \rightarrow T$ and $T_{n}^{*} \rightarrow T^{*}$ pointwise and both $\left\{T_{n}\right\}$ and $\left\{T_{n}^{*}\right\}$ are collectively compact.

We remark again that if $X=H$ is a Hilbert space, we then let $T^{*}$ and $T_{n}^{*}$ denote 
the Hilbert adjoints. In the proof of Theorem 2 in this context we would let $\phi_{1}$, $\ldots, \phi_{m}$ be an orthonormal basis for $R(E)$ and let $\phi_{j}^{*}=E^{*} \phi_{j}$. Then $\phi_{1}^{*}, \ldots, \phi_{m}^{*} \in$ $N\left(\left(\bar{\mu}-T^{*}\right)^{\alpha}\right)$ and $\operatorname{trace}\left(\hat{T}-\hat{T}_{n}\right)=\sum_{j=1}^{m}\left(\left(\hat{T}-\hat{T}_{n}\right) \phi_{j}, \phi_{j}^{*}\right)$, where $(\cdot ;)$ denotes the inner product on $H$, and with only minor modifications all the results of this section are valid.

Finally, we note that if we were given a family $\left\{T_{h}\right\}_{0<n \leqslant 1}$ of compact operators such that $T_{h} \rightarrow T$ pointwise as $h \rightarrow 0$ and $\left\{T_{h}\right\}_{0<h \leqslant 1}$ is collectively compact, or $T_{h} \rightarrow T$ in norm, then all of the results in this section (with certain obvious modifications) hold.

4. Applications. We outline in this section several applications of the results in Section 3.

(a) We consider first the approximation of eigenvalues of nonselfadjoint 2 nd order elliptic partial differential operators by Galerkin-type methods.

Let $\Omega$ be a bounded domain in $R^{N}$ with boundary $\partial \Omega$ which will be assumed to be of class $C^{\infty}$. $H^{s}(\Omega)$, for any real $s \geqslant 0$, will denote the usual Sobolev space and the norm on $H^{s}$ will be denoted by $\|\cdot\|_{s} \cdot H^{0}(\Omega)=L_{2}(\Omega)$ and the inner product on $L_{2}$ will be denoted by $(\cdot ;) . H_{0}^{1}(\Omega)$ is the subspace of $H^{1}(\Omega)$ consisting of those functions which vanish on $\partial \Omega$.

Let $L$ be defined by

$$
L u=-\sum_{i, j=1}^{N} \frac{\partial}{\partial x_{i}}\left(a_{i j} \frac{\partial u}{\partial x_{j}}\right)+\sum_{i=1}^{N} b_{i} \frac{\partial u}{\partial x_{i}}+c u
$$

where $a_{i j}, b_{i}$ and $c$ are in $C^{\infty}(\bar{\Omega})$. We assume $L$ is uniformly strongly elliptic, i.e., there is a constant $a_{0}>0$ such that

$$
\operatorname{Re} \sum_{i, j=1}^{N} a_{i j}(x) \xi_{i} \xi_{i} \geqslant a_{0} \sum_{i=1}^{N} \xi_{i}^{2}
$$

for all real $\xi_{1}, \ldots, \xi_{N}$ and $x \in \Omega$. Associated with $L$ is the sesquilinear form on $H^{1}(\Omega)$ defined by

$$
B(u, v)=\sum_{i, j=1}^{N} \int_{\Omega} a_{i j} \frac{\partial u}{\partial x_{i}} \frac{\partial \bar{v}}{\partial x_{j}} d x+\sum_{i=1}^{N} \int_{\Omega} b_{i} \frac{\partial u}{\partial x_{i}} \bar{v} d x+\int_{\Omega} c u \bar{v} .
$$

Let $b=\max _{1 \leqslant i \leqslant N ; x \in \Omega}\left|b_{i}(x)\right|$. We assume without loss that $\operatorname{Re} c \geqslant a_{0} / 2+b^{2} / 2 a_{0}$ since adding a constant to $c$ only shifts the eigenvalues. Under this assumption $B$ is coercive on $H^{1}(\Omega)$, i.e.,

$$
\operatorname{Re} B(u, u) \geqslant 1 / 2 a_{0}\|u\|_{1}^{2}
$$

for all $u \in H^{1}(\Omega)$. The boundedness of the coefficients of $L$ implies that $B$ is continuous on $H^{1}(\Omega)$, i.e., there is a constant $a_{1}$ such that

$$
|B(u, v)| \leqslant a_{1}\|u\|_{1}\|v\|_{1}
$$

for all $u, v \in H^{1}(\Omega)$.

Given a closed subspace $V$ of $H^{1}(\Omega)$ with $H_{0}^{1}(\Omega) \subset V$ we can formulate a boundary value problem associated with $B$ as follows: Given $f \in L_{2}(\Omega)$ find $u \in V$ such that 


$$
B(u, \phi)=(f, \phi)
$$

for all $\phi \in V$. Under our assumptions this problem always has a unique solution which we denote by $T f$. If $V=H_{0}^{1}(\Omega)$ this is the weak formulation of the Dirichlet problem associated with $L$ and if $V=H^{1}(\Omega)$ it is the Neumann problem. By the Rellich compactness theorem $T$ is a compact operator on $L_{2}(\Omega)$. We now suppose $V$ is either $H_{0}^{1}(\Omega)$ or $H^{1}(\Omega)$. In addition, $T$ satisfies the following regularity estimate:

$$
\|T f\|_{s+2} \leqslant C_{s}\|f\|_{s}
$$

for all $f \in H^{s}(\Omega)$ for some constant $C_{s}$ (cf. [4]). The $L_{2}(\Omega)$-adjoint $T^{*}$ of $T$ satisfies $B\left(\phi, T^{*} \psi\right)=(\phi, \psi)$ for all $\phi \in V$ and, as for $T$, the regularity estimate

$$
\left\|T^{*} f\right\|_{s+2} \leqslant C_{s}\|f\|_{s}
$$

for all $f \in H^{s}(\Omega)$.

An eigenvalue corresponding to this boundary value problem is a complex number $\lambda$ such that

$$
B(u, \phi)=\lambda(u, \phi)
$$

for some nonzero $u \in V$ and all $\phi \in V$. Clearly $\lambda$ is an eigenvalue satisfying (4.6) if and only if $\mu=1 / \lambda$ is an eigenvalue of $T$. If $\lambda$ and $u$ satisfy (4.6), then, because of the smoothness assumptions on $\partial \Omega$ and the coefficients of $L, L u=\lambda u$ in $\Omega$ and $u=$ $0[\partial u / \partial \nu=0]$ on $\partial \Omega$ if $V=H_{0}^{1}(\Omega)\left[V=H^{1}(\Omega)\right]$ where $\partial / \partial \nu$ is the conormal derivative.

Let $\left\{S_{h}\right\}_{0<n \leqslant 1}$ be a one-parameter family of finite dimensional spaces. For given integers $k$ and $r$ with $0 \leqslant k \leqslant r$ we say that $\left\{S_{h}\right\}_{0<h \leqslant 1}$ is of class $S_{k, r}$ if $S_{h} \subset$ $H^{k}(\Omega)$ for each $h$ and if there is a constant $C$ independent of $h$ and $v$ such that

$$
\inf _{\chi \in S_{h}} \sum_{j=0}^{k} h^{j}\|v-\chi\|_{j} \leqslant C h^{t}\|v\|_{t}
$$

for all $0<h \leqslant 1$ and $v \in H^{t}(\Omega)$ with $k \leqslant t \leqslant r$. We define the class $\stackrel{\circ}{S}_{k, r}$ to consist of those families $\left\{S_{h}\right\}_{0<n \leqslant 1}$ such that $S_{h} \subset H^{k}(\Omega)$ for each $h$ and (4.7) is required to hold only for $v \in H^{t}(\Omega) \cap H_{0}^{1}(\Omega)$. Many examples of such families have been studied recently. Each of the usual sets of trial functions used in the finite element method is of class $S_{k, r}\left[S_{k, r}\right]$ for some $k$ and $r$.

If $V=H^{1}(\Omega)$ we assume we are given a family $\left\{S_{h}\right\}$ of class $S_{1, r}$ with $r \geqslant 2$; if $V=H_{0}^{1}(\Omega)$ we assume we are given a family of class $\stackrel{\circ}{S}_{1, r}$ with $r \geqslant 2$ and assume, in addition, that $S_{h} \in H_{0}^{1}(\Omega)$ for each $h$. We now consider the approximate eigenvalues defined by the Galerkin method with trial functions given by $\left\{S_{h}\right\}$, i.e., the complex numbers $\lambda(h)$ satisfying $B(w, \phi)=\lambda(h)(w, \phi)$ for some nonzero $w \in S_{h}$ and all $\phi \in S_{h}$. These will be approximations to the eigenvalues of (4.6). For $f \in L_{2}(\Omega)$ let $T_{h} f$ be the unique element in $S_{h}$ such that

$$
B\left(T_{h} f, \phi\right)=(f, \phi)
$$

for all $\phi \in S_{h}$. It is easily seen that $T_{h} w=(1 / \lambda(h)) w$. Thus the eigenvalues $\mu(h)$ of $T_{h}$ are reciprocals of the Galerkin eigenvalues $\lambda(h)$. The eigenvalues of (4.6) are thus char- 
acterized as the eigenvalues of the compact operator $T$ and the approximate eigenvalues are characterized as the eigenvalues of the compact operators $T_{n}$.

In order to apply the results of Section 3 , we need to obtain estimates for $T-T_{h}$. Let $f \in H^{t-2}(\Omega)$ for some $t$ with $2 \leqslant t \leqslant r$. From (4.3) and (4.8) we have $B\left(\left(T-T_{h}\right) f, \phi\right)=0$ for all $\phi \in S_{h}$. Hence, using (4.1),

$$
\begin{aligned}
\left\|\left(T-T_{h}\right) f\right\|_{1}^{2} & \leqslant \frac{2}{a_{0}}\left|B\left(\left(T-T_{h}\right) f,\left(T-T_{h}\right) f\right)\right| \\
& =\frac{2}{a_{0}}\left|B\left(\left(T-T_{h}\right) f, T f-\chi\right)\right|
\end{aligned}
$$

for any $\chi \in S_{h}$. Thus, using (4.2), (4.7) and (4.4) we have

$$
\left\|\left(T-T_{h}\right) f\right\|_{1} \leqslant C \inf _{\chi \in S_{h}}\|T f-\chi\|_{1} \leqslant C h^{t-1}\|T f\|_{t} \leqslant C h^{t-1}\|f\|_{t-2}
$$

for an appropriate constant $C$. Now let $\psi \in H^{s}$ with $0 \leqslant s \leqslant r-2$. Then

$$
\left(\left(T-T_{h}\right) f, \psi\right)=B\left(\left(T-T_{h}\right) f, T^{*} \psi\right)=B\left(\left(T-T_{h}\right) f, T^{*} \psi-\chi\right)
$$

for any $\chi \in S_{h}$. Thus, using (4.2) and (4.9), we have

$$
\left|\left(\left(T-T_{h}\right) f, \psi\right)\right| \leqslant C h^{t-1}\|f\|_{t-2} \inf _{\chi \in S_{h}}\left\|T^{*} \psi-\chi\right\|_{1} .
$$

Using (4.7) and (4.5), we get

$$
\left|\left(\left(T-T_{h}\right) f, \psi\right)\right| \leqslant C h^{t+s}\|f\|_{t-2}\|\psi\|_{s} .
$$

The argument leading to (4.10) is due to Nitsche [13].

From the estimate (4.10) we can obtain all of the estimates needed to apply the results of Section 3. (4.10) with $t=2$ and $s=0$ yields $\left|\left(\left(T-T_{h}\right) f, \psi\right)\right| \leqslant$ $C h^{2}\|f\|_{0}\|\psi\|_{0}$, and hence

$$
\left\|\left(T-T_{h}\right) f\right\|_{0}=\sup _{\psi \in L_{2}(\Omega),\|\psi\|_{0}=1}\left|\left(\left(T-T_{h}\right) f, \psi\right)\right| \leqslant C h^{2}\|f\|_{0}
$$

for all $f \in L_{2}(\Omega)$. This shows that $T_{h} \rightarrow T$ in norm, and hence that all the results of Section 3 apply. Now we estimate $\left\|\left.\left(T-T_{h}\right)\right|_{R(E)}\right\|$. Toward this end let $f \in R(E)$.

Under our assumptions the eigenvectors and generalized eigenvectors of $T$ (or $L$ ) are in $H^{t}(\Omega)$ for any $t \geqslant 0$. Thus from (4.10) with $t=r$ and $s=0$ we get

$$
\left|\left(\left(T-T_{h}\right) f, \psi\right)\right| \leqslant C h^{r}\|f\|_{r-2}\|\psi\|_{0}
$$

and hence

$$
\begin{aligned}
\left\|\left.\left(T-T_{h}\right)\right|_{R(E)}\right\| & =\sup _{f \in R(E), \psi \in L_{2}(\Omega) ;\|f\|_{0}=\|\psi\|_{0}=1}\left|\left(\left(T-T_{h}\right) f, \psi\right)\right| \\
& \leqslant C h^{r} \sup _{f \in R(E),\|f\|_{0}=1}\|f\|_{r-2} \leqslant C h^{r} .
\end{aligned}
$$

Next we estimate $\left\|\left.\left(T^{*}-T_{h}^{*}\right)\right|_{R\left(E^{*}\right)}\right\|$. The generalized eigenfunctions of $T^{*}$ are also in $H^{t}$ for any $t \geqslant 0$. Thus, using (4.10) with $s=r-2$ and $t=2$, we find

$$
\left|\left(f,\left(T^{*}-T_{h}^{*}\right) \psi\right)\right|=\left|\left(\left(T-T_{h}\right) f, \psi\right)\right| \leqslant C h^{r}\|f\|_{0}\|\psi\|_{r-2}
$$


which yields

$$
\left\|\left.\left(T^{*}-T_{h}^{*}\right)\right|_{R\left(E^{*}\right)}\right\| \leqslant C h^{r} .
$$

Finally we consider $\Sigma_{j=1}^{m}\left|\left(\left(T-T_{n}\right) \phi_{j}, \phi_{j}^{*}\right)\right|$. It follows immediately from (4.10) with $t=r$ and $s=r-2$ that

$$
\sum_{j=1}^{m}\left|\left(\left(T-T_{h}\right) \phi_{j}, \phi_{j}^{*}\right)\right| \leqslant C h^{2 r-2} .
$$

Let $\lambda$ be an eigenvalue of algebraic multiplicity $m$ satisfying (4.6) and let $\mu=1 / \lambda$. Then the eigenvalues $\mu_{1}(h), \ldots, \mu_{m}(h)$ which converge to $\mu$ as $h \rightarrow 0$ are computed as the reciprocals of certain eigenvalues $\lambda_{1}(h), \ldots, \lambda_{m}(h)$ of the finite dimensional eigenvalue problem

$$
B(w, \phi)=\lambda_{j}(h)(w, \phi),
$$

for nonzero $w \in S_{h}$ and all $\phi \in S_{h}$. Thus using (4.11), (4.12) and (4.13) we see that Theorem 3 yields the estimate

$$
\left|\lambda-\left(\frac{1}{m} \sum_{j=1}^{m} 1 / \lambda_{j}(h)\right)^{-1}\right| \leqslant C h^{2 r-2} .
$$

Using Theorem 1 we have

$$
\hat{\delta}\left(R(E), R\left(E_{n}\right)\right) \leqslant C h^{r}
$$

for the generalized eigenvectors.

The estimates (4.14) and (4.15) were obtained by Bramble and Osborn [7]. The results of Section 3 also apply to several other methods for constructing approximations to the eigenvalues of the eigenvalue problem associated with $L$ and the Dirichlet boundary conditions: the least squares method of Bramble and Schatz [8], methods of Nitsche [16], [17], and the Lagrange multiplier method of Babuska [3]. In addition, the results of Section 3 apply to the approximation of Steklov eigenvalues as studied in [6]. For a more complete discussion of all of these methods, see [7].

(b) Let $(T f)(x)=\int_{0}^{1} K(x, y) f(y) d y$ where $K(x, y)$ is continuous for $0 \leqslant x, y \leqslant 1$. $T$ is a compact operator on $C[0,1]$-the Banach space of continuous functions with the supremum norm. Let $\left\{T_{n}\right\}$ be a sequence of approximations to $T$ defined by numerical quadrature, i.e., let

$$
\left(T_{n} f\right)(x)=\sum_{j=1}^{n} w_{n j} K\left(x, y_{n_{j}}\right) f\left(y_{n_{j}}\right),
$$

where $0 \leqslant y_{n_{j}} \leqslant 1$ and the weights $w_{n_{j}}$ are real or complex and we assume that $\sum_{j=1}^{n} w_{n_{j}} g\left(y_{n_{j}}\right) \rightarrow \int_{0}^{1} g(x) d x$ for each $g \in C[0,1]$. Then it is known (cf. [1]) that $T_{n} \rightarrow T$ pointwise and $\left\{T_{n}\right\}$ is collectively compact.

Thus Theorems 1, 2, 5 and 6 apply to this type of approximation. A complete discussion of approximation by numerical quadrature can be found in [1] ; compare also [2].

(c) Let $a(x)$ and $c(x)$ be periodic functions of period 1 which are defined by 


$$
a(x)=\left\{\begin{array}{ll}
p_{1}, & -1 / 2 \leqslant x<0, \\
p_{2}, & 0 \leqslant x<1 / 2,
\end{array} \quad c(x)= \begin{cases}q_{1}, & -1 / 2 \leqslant x<0, \\
q_{2}, & 0 \leqslant x<1 / 2,\end{cases}\right.
$$

where $p_{1}, p_{2}, q_{1}, q_{2}$ are positive constants, and let $a_{h}(x)=a(x / h)$ and $c_{h}(x)=c(x / h)$ where $h$ is a small parameter. Recently Babuška [4], [5] has studied the eigenvalue problem,

$$
\frac{d}{d x}\left(a_{h} \frac{d u_{h}}{d x}\right)=\lambda_{h} c_{h} u_{h}, \quad 0 \leqslant x \leqslant 1
$$

together with the boundary conditions,

$$
u(0)=u(1)=0
$$

or

$$
u(0)=d u(1) / d x=0 .
$$

As $h \rightarrow 0$ the problem (4.16), (4.17) [(4.18)] "tends" to the "homogenized" problem,

$$
\frac{d}{d x}\left(a_{0} \frac{d u}{d x}\right)=\lambda_{0} c_{0} u, \quad 0 \leqslant x \leqslant 1,
$$

where $a_{0}^{-1}=1 / 2\left(p_{1}^{-1}+p_{2}^{-1}\right)$ and $c_{0}=1 / 2\left(q_{1}+q_{2}\right)$, together with the boundary conditions (4.17) $[(4.18)]$. The problem (4.19), (4.17) [(4.18)] is considered as an approximation to (4.16), (4.17) [(4.18)] for $h$ small.

Computational experiments show that with the boundary conditions (4.17) the eigenvalue error is proportional to $h^{2}$, whereas with (4.18) the eigenvalue error is proportional to $h$. The higher order convergence with the boundary condition (4.17) can be explained as follows.

Let $T$ and $T_{h}$ be the inverses of the differential operators defined by (4:16), (4.17) and (4.19), (4.17), respectively. The eigenvalue problems (4.16), (4.17) and (4.19), (4.17) are selfadjoint and for their first eigenvalues $\lambda_{h}^{1}$ and $\lambda_{0}^{1}$, we have from Theorem 3 the estimate

$$
\left|\lambda_{0}^{1}-\lambda_{h}^{1}\right| \leqslant C\left\{\left(\left(T-T_{n}\right) \phi, \phi\right)+\left\|\left(T-T_{n}\right) \phi\right\|_{0}^{2}\right\},
$$

where $\phi$ is the unit eigenvector of (4.19), (4.17) corresponding to $\lambda_{0}^{1}$. Since $\phi \in$ $H^{2}[0,1]$, we will have $\left\|\left(T-T_{h}\right) \phi\right\|_{0} \leqslant C h$. Now Babuska has shown that $\left(T-T_{h}\right) \phi$ can be expressed in the form $\left(T-T_{h}\right) \phi=h^{2} u+h v$ where $v$ is odd with respect to $x=1 / 2$. Since $\phi$ is a multiple of $\sin x$, we see that $(v, \phi)=0$ and thus $\left(\left(T-T_{h}\right) \phi, \phi\right)=$ $h^{2}(u, \phi)$. Hence from (4.20) we have $\left|\lambda_{0}^{1}-\lambda_{h}^{1}\right| \leqslant C h^{2}$.

It follows from Theorem 1 that the eigenvector error is of the order $h$ for both types of boundary conditions. Thus, with this type of approximation, the eigenvalue error is not always of higher order than the eigenvector error.

(d) Given a compact operator $T$, a common way to define an approximating sequence of operators is to let $T_{n}=P_{n} T$ where $\left\{P_{n}\right\}$ is a sequence of projections which converge pointwise to the identity. Then $T_{n} \rightarrow T$ in norm.

Thus Theorems 1, 3, 4 and 5 apply to such projection methods. We limit ourselves to pointing out that the estimate in Theorem 2 yields 


$$
\begin{aligned}
|\mu-\hat{\mu}(n)| & \leqslant \sum_{j=1}^{m}\left|\left[\left(T-P_{n} T\right) \phi_{j}, \phi_{j}^{*}\right]\right|+C_{3}\left\|\left.\left(T-T_{n}\right)\right|_{R(E)}\right\|\left\|\left.\left(T^{*}-T_{n}^{*}\right)\right|_{R\left(E^{*}\right)}\right\| \\
& =\sum_{j=1}^{m}\left|\left[\left(I-P_{n}\right)^{2} T \phi_{j}, \phi_{j}^{*}\right]\right|+C_{3}\left\|\left.\left(T-T_{n}\right)\right|_{R(E)}\right\|\left\|\left.\left(T^{*}-T_{n}^{*}\right)\right|_{R\left(E^{*}\right)}\right\| \\
& \leqslant \sum_{j=1}^{m}\left\|\left(T-T_{n}\right) \phi_{j}\right\|\left\|\left(I-P_{n}^{*}\right) \phi_{j}^{*}\right\|+C_{3}\left\|\left.\left(T-T_{n}\right)\right|_{R(E)}\right\|\left\|\left.\left(T^{*}-T_{n}^{*}\right)\right|_{R\left(E^{*}\right)}\right\|
\end{aligned}
$$

Department of Mathematics

University of Maryland

College Park, Maryland 20742

1. P. M. ANSELONE, Collectively Compact Operator Approximation Theory, Prentice-Hall, Englewood Cliffs, N. J., 1971.

2. K. ATKINSON, "Convergence rates for approximate eigenvalues of compact integral operators," SIAM J. Numer. Anal., v. 12, 1975, pp. 213-222.

3. I. BABUŠKA, "The finite element method with Lagrangian multipliers," Numer. Math., v. 20, 1973, pp. 179-192.

4. I. BA BUŠKA, Solution of Interface Problems by Homogenization. I, Technical Note BN-782, Institute for Fluid Dynamics and Applied Mathematics, University of Maryland, College Park, Md., 1974.

5. I. BABUŠKA, Solution of Problems with Interfaces and Singularities, Proc. Sympos., Math. Res. Center, University of Wisconsin, Madison, Wis., April 1974.

6. J. H. BRAMBLE \& J. E. OSBORN, "Approximation of Steklov eigenvalues of nonselfadjoint second order elliptic operators," The Mathematical Foundations of the Finite Element Method with Applications to Partial Differential Equations (edited by A. K. Aziz), Academic Press, New York, 1972, pp. 387-408.

7. J. H. BRAMBLE \& J. E. OSBORN, "Rate of convergence estimates for nonselfadjoint eigenvalue approximations," Math. Comp., v. 27, 1973, pp. 525-549.

8. J. H. BRAMBLE \& A. H. SCHATZ, "Rayleigh-Ritz-Galerkin methods for Dirichlet's problem using subspaces without boundary conditions," Comm. Pure Appl. Math., v. 23; 1970, pp. 653-675. MR 42 \#2690.

9. N. DUNFORD \& J. T. SCHWARTZ, Linear Operators. II: Spectral Theory. Selfadjoint Operators in Hilbert Space, Interscience, New York, 1963. MR 32 \#6181.

10. T. KATO, "Perturbation theory for nullity, deficiency and other quantities of linear operators," J. Analyse Math., v. 6, 1958, pp. 261-322. MR 21 \#6541.

11. T. KATO, Perturbation Theory for Linear Operators, Die Grundlehren der Math. Wissenschaften, Band 132, Springer-Verlag, New York, 1966. MR 34 \#3324.

12. H. KREISS, "Difference approximations for boundary and eigenvalue problems for ordinary differential equations," Math. Comp., v. 26, 1972, pp. 605-624.

13. D. C. LAY, Private communication.

14. J.-L. LIONS \& E. MAGENES, Problèmes aux limites non homogènes et applications. Vol. I, Travaux et Recherches Mathématiques, no. 17, Dunod, Paris, 1968. MR 40 \#512.

15. J. NITSCHE, "Ein Kriterium für die Quasi-Optimalität des Ritzschen Verfahrens," Numer. Math., v. 11, 1968, pp. 346-348. MR 38 \#1823.

16. J. NITSCHE, "Über ein Variationsprinzip zur Lösung von Dirichlet-Problemen bei Verwendung von Teilräumen, die keinen Randbedingungen unterworfen sind," Abh. Math. Sem. Univ. Hamburg, v. 36, 1970/71.

17. J. NITSCHE, "A projection method for Dirichlet-problems using subspaces with nearly zero boundary conditions." (Preprint.)

18. G. M. VAINIKKO, "Asymptotic error bounds for projection methods in the eigenvalue problem," Ž. Vycisl. Mat. i Mat. Fiz., v. 4, 1964, pp. 405-425=U. S. S. R. Comput. Math. and Math. Phys., v. 4, 1964, pp. 9-36. MR 31 \#615.

19. G. M. VAIINIKKO, "On the rate of convergence of certain approximation methods of Galerkin type in an eigenvalue problem," Izv. Vyss. UCebn. Zaved. Matematika, v. 1966, no. 2(51), pp. 37-45; English transl., Amer. Math. Soc. Transl. (2), v. 86, 1970, pp. 249-259. MR 33 \#6824; 41 \#1462.

20. G. M. VAĬNIKKO, "Rapidity of convergence of approximation methods in the eigenvalue problem," Ž. Vyčisl. Mat. i Mat. Fiz., v. 7, 1967, pp. 977-987=U. S. S. R. Comput. Math. and Math. Phys., v. 7, 1967, pp. 18-32. MR $36 \# 4798$. 